\title{
Sonolência excessiva diurna na população geral de um município brasileiro
}

Excessive daytime sleepiness in the general population of Brazilian city

José Carlos Souza', Luis Alberto Magna², Sandra Aiache, Natália Segaglio Magna ${ }^{4}$

\section{RESUMO}

Objetivo: Buscou-se avaliar a prevalência da sonolência excessiva diurna (SED) na população geral de um município brasileiro. Método: Foram feitas 198 entrevistas domiciliares entre os adultos, em amostra representativa da população geral de Ribeirão do Largo, BA. A amostragem foi aleatória simples. Tinham SED as pessoas com 11 ou mais pontos na Escala de Sonolência de Epworth (ESE). Usaram-se os testes de qui-quadrado, Fisher e ANOVA; nível

\section{Palavras-chave}

Sonolência,

Escala de Sonolência Epworth, Ribeirão do Largo.

\section{Key-words}

Sleepiness, Epworth

Sleepiness Scale,

Ribeirão do Largo. de significância 5\%. Resultados: Tinham SED 21,5\% da população (DP = 2,9\%; IC 15,8\% a 27,2\%); não houve associação significativa entre SED e idade ( $p=0,924)$, nem IMC ( $p=0,197)$, sexo $(p=0,095)$, instrução $(p=0,700)$, estado civil $(p=0,414)$ e uso de hipnóticos $(p=0,176)$. Houve associação com o despertar precoce $(p=0,046)$. Conclusão: Foi alta a prevalência de SED na população estudada.

\section{ABSTRACT}

Objective: To assess the prevalence of excessive daytime sleepiness (ESD) in general population of a Brazilian city. Methods: It was determined by means of 198 home interviews of adults, in a representative sample of Ribeirão do Largo city, Brazil. The random sample was simple. ESD was considered in those with index 11 or more in the Epworth Sleepiness Scale (ESS). Statistics used chi-square, Fisher and ANOVA; the significance level was 5\%. Results: The prevalence of ESD was $21.5 \%$ of population (SD $=2.9 \%$; Cl 15.8\%-27.2\%). No significant association was found between ESD and age ( $p=0.924)$, nor with body mass index ( $p=0.197)$, sex $(p=0.095)$, schooling $(p=0,700)$, marital status ( $p=0.414)$ and the use of hypnotics $(p=0.176)$. There was significant association with early awakening $(p=0.046)$. Conclusion: There was a high prevalence of ESD in the population.
1 Universidade Católica Dom Bosco (UCDB).

2 Departamento de Genética Médica da Universidade Estadual de Campinas (UNICAMP).

3 Universidade Estadual de Ciências de Saúde de Alagoas.

4 Faculdade de Educação Física da Pontifícia Universidade Católica de Campinas (PUCCAMP).

Endereço para correspondência: José Carlos Souza

Rua Theotônio Rosa Pires, 88, Vila Rosa Pires - 79004-340 - Campo Grande, MS

E-mail: josecarlossouza@uol.com.br 


\section{INTRODUÇÃO}

A sonolência excessiva diurna (SED) é o segundo transtorno do sono mais comum na população geral, depois apenas da insônial ${ }^{1-11}$; sua prevalência varia de 0,3\% a 13,3\% 5,12,13. Afeta as funções cognitivas, como memória e concentração, e a qualidade de vida, constituindo-se preocupante problema de saúde pública7-11,14. Sua etiologia varia desde transtornos mentais, como a depressão; endócrinos, como a obesidade; e respiratórios, como a apnéia do sono, entre outros ${ }^{15-18}$.

O objetivo deste estudo foi detectar a prevalência da SED na população geral de Ribeirão do Largo, BA. Por se tratar de um estudo epidemiológico, não houve a intenção de se detectar as possíveis etiologias da SED, até mesmo porque o instrumento utilizado não se propõe a tal. Este faz parte de uma série de pesquisas dos autores sobre a prevalência da SED em diversas cidades brasileiras.

\section{MÉTODO}

Realizou-se um estudo epidemiológico, descritivo, analítico e de corte transversal na área urbana do município de Ribeirão do Largo, na região sudoeste do Estado da Bahia, Brasil. Conforme o Instituto Brasileiro de Geografia e Estatística (IBGE), o município tem uma população geral de 15.303 habitantes, dos quais 4.437 residem na área urbana. Foram utilizados a Escala de Sonolência de Epworth (ESE) e um questionário sociodemográfico de fácil compreensão. A ESE se propõe apenas a dar um indicativo da presença de SED na população, e não de suas etiologias. Utilizou-se a técnica de entrevistas pessoais nos lares, com uma amostra aleatória simples de 198 sujeitos, de ambos os sexos, com idade de 18 anos ou mais, entre os dias 14 e 20 de janeiro de 2001.

Participaram da coleta de dados cinco entrevistadores devidamente treinados. Todos os entrevistados assinaram (quando analfabetos carimbaram as suas impressões digitais) um termo de consentimento livre e esclarecido. Não houve rejeição. Houve a aprovação do Comitê de Ética em Pesquisa da Universidade Católica Dom Bosco (UCDB), Campo Grande, MS. A análise estatística foi descritiva e realizada com os testes $t$ de Student, exato de Fisher, análise de variância posterior por LSD (least squares difference). Quando necessário, a comparação de médias foi complementada pelos métodos de Kruskal-Wallis e Mann-Whitney, respectivamente.

Foram consideradas as variáveis sociodemográficas idade, sexo, grau de instrução, estado civil e índice de massa corporal (IMC); a SED, com base na ESE (escore total inferior a 11 significando ausência de SED e totalizando 11 ou mais presença de SED); uso de hipnóticos, dificuldade para dormir, acordar a noite e acordar mais cedo que o desejado.
Os métodos estatísticos empregados incluíram estatística descritiva, com medidas de tendência central e de variabilidade, com determinação do intervalo de confiança de 95\%; e estatística analítica, com comparação de médias de grupos independentes avaliada pelos testes $t$ de Student e não-paramétrico de Mann-Whitney. A verificação de associação em tabelas de contingência se fez pelo teste qui-quadrado, complementado pelo teste exato de Fisher, quando presentes baixas freqüências esperadas segundo a hipótese nula. Em todos os casos, adotou-se o nível de significância de 5\% ( $p<0,05)$.

Utilizou-se para o cálculo estatístico o pacote SPSS (SPSS 16.0 para Windows, 2007, SPSS Inc., EUA).

\section{RESULTADOS}

Segundo o escore total da ESE, somente quatro sujeitos pontuaram 16 ou mais; assim, visando à maior precisão no estudo analítico, foi incluída juntamente a categoria de sujeitos com sonolência excessiva diurna (SED). Assim, 21,5\% dos sujeitos da população-alvo têm SED (DP = 2,9\%; IC 95\% $15,8 \%$ a $27,2 \%)$.

Não houve diferença significativa entre as médias de idade dos sujeitos sem e com SED ( $p=0,924)$. Também a média do IMC é semelhante nos dois grupos $(p=0,197)$. Quanto ao sexo, instrução e estado civil, nenhuma dessas três variáveis influenciou a categorização dos sujeitos segundo a ESE $(p=0,095 ; p=0,700$; e $p=0,414$, respectivamente).

Foram feitas questões relacionadas à insônia, segundo os critérios do DSM-IV'19, como dificuldade para adormecer e incômodo para tal e sua duração, freqüência de despertar noturno e tempo de incômodo, como também o despertar precoce e tempo de incômodo para tal, além da presença ou não de prejuízo no dia seguinte e o uso de hipnótico.

Não houve associação significativa entre a dificuldade para dormir e a divisão segundo a ESE $(p=0,719)$ (Tabela 1). O incômodo para começar a dormir também não se associou com a divisão dos sujeitos segundo a ESE $(p=0,836)$. Não se observou, também, associação entre o tempo de incômodo da dificuldade para começar a dormir e a divisão pela ESE $(p=0,964)$. Não houve associação entre a freqüência de acordar à noite no último mês e a divisão pela ESE $(p=0,215)$. $O$ tempo de incômodo por ter acordado à noite não influenciou significativamente a divisão dos sujeitos consoante a $\operatorname{ESE}(p=0,287)$.

Houve uma associação significativa entre o acordar mais cedo que o desejado e a divisão segundo a ESE $(p=0,046)$, o que ocorre porque, entre os sujeitos que acordam uma ou duas vezes por semana, há maior proporção de incidência de SED do que nas demais categorias, que se assemelham $(p=0,334)$. 
Tabela 1. Relação entre SED e dificuldade para dormir, acordar a noite e acordar mais cedo

\begin{tabular}{|c|c|c|c|c|c|c|c|}
\hline \multirow[b]{2}{*}{ Dificuldade para dormir } & \multicolumn{2}{|c|}{ Sem SED } & \multicolumn{2}{|c|}{ SED ou apnéia } & \multicolumn{2}{|c|}{ Total } & \multirow[t]{2}{*}{$p$} \\
\hline & $\mathrm{n}$ & $\%$ & $\mathrm{n}$ & $\%$ & $\mathrm{n}$ & $\%$ & \\
\hline Não teve & 97 & 78,86 & 26 & 21,14 & 123 & 100,00 & \\
\hline Menos de 1 vez/semana & 11 & 84,62 & 2 & 15,38 & 13 & 100,00 & 0,719 \\
\hline 1 ou 2 vezes/semana & 16 & 69,57 & 7 & 30,43 & 23 & 100,00 & \\
\hline \multirow[t]{2}{*}{3 ou mais vezes/semana } & 29 & 78,38 & 8 & 21,62 & 37 & 100,00 & \\
\hline & \multicolumn{2}{|c|}{ Sem SED } & \multicolumn{2}{|c|}{ SED ou apnéia } & \multicolumn{2}{|r|}{ Total } & $p$ \\
\hline Acordou à noite & $n$ & $\%$ & $n$ & $\%$ & $\mathrm{n}$ & $\%$ & \\
\hline Não acordou & 92 & 82,14 & 20 & 17,86 & 112 & 100,00 & \\
\hline Menos de 1 vez/semana & 20 & 80,00 & 5 & 20,00 & 25 & 100,00 & 0,215 \\
\hline 1 ou 2 vezes/semana & 17 & 73,91 & 6 & 26,09 & 23 & 100,00 & \\
\hline \multirow[t]{2}{*}{3 ou mais vezes/semana } & 23 & 65,71 & 12 & 34,29 & 35 & 100,00 & \\
\hline & \multicolumn{2}{|c|}{ Sem SED } & \multicolumn{2}{|c|}{ SED ou apnéia } & \multicolumn{2}{|c|}{ Total } & $\mathrm{p}$ \\
\hline Frequiência de acordar mais cedo & $n$ & $\%$ & $n$ & $\%$ & $\mathrm{n}$ & $\%$ & \\
\hline Não acordou mais cedo & 112 & 82,96 & 23 & 17,04 & 135 & 100,00 & \\
\hline Menos de 1 vez/semana & 14 & 73,68 & 5 & 26,32 & 19 & 100,00 & 0,046 \\
\hline 1 ou 2 vezes/semana & 12 & 57,14 & 9 & 42,86 & 21 & 100,00 & \\
\hline 3 ou mais vezes/semana & 15 & 71,43 & 6 & 28,57 & 21 & 100,00 & \\
\hline
\end{tabular}

O tempo de incômodo por acordar mais cedo que o desejado não se associou com a divisão pela ESE $(p=0,073)$; no entanto, pode-se discutir eventualmente a tendência de maior proporção de SED entre aqueles com mais de um ano de ocorrência em relação aos demais. O prejuízo no dia seguinte não se associou significativamente com a ESE $(p=0,413)$.

A utilização de remédio no último mês se dá independentemente de ocorrência de SED $(p=0,176)$, o uso de remédio durante toda a vida do sujeito também não mostrou associação com a divisão conforme a ESE $(p=0,139)$.

\section{DISCUSSÃO}

A prevalência de SED de 21,5\%, detectada neste estudo, é semelhante a vários índices observados na população geral. Janson et al."1 referem 14,7\% nos países do norte europeu; Ohayon et al. ${ }^{20}$, 20,7\% no Reino Unido; Hara ${ }^{8}$, 16,8\% em Bambuí, MG; e Souza; Reimão; Magna ${ }^{21}$, 18,9\% em Campo Grande, MS. Outros índices menores foram encontrados, sendo 9,8\% na Finlândia10, 9\% na Finlândia9 e 6,7\% na Califórnia, EUA $A^{15}$.

Não houve correlações significativas entre a SED e a idade, o IMC, o sexo, a instrução, o estado civil e o uso de hipnóticos. Outros estudos corroboram com essa falta de correlação ${ }^{2,17,20-22}$. Supõe-se que isso seja em razão da larga variedade de causas da SED.
A SED pode estar associada a vários distúrbios, incluindo a insônia, a depressão, a síndrome da apnéia obstrutiva do sono, a narcolepsia e o uso de drogas hipnótica 16,19,22.

Neste estudo, houve correlação da SED com o distúrbio do despertar precoce. Hublin et al. ${ }^{9}$ detectaram, entre as pessoas com SED, 20,7\% das mulheres e $28,6 \%$ dos homens com insônia. Ohayon et al. ${ }^{20}$ verificaram que os grupos com SED grave e moderada tinham maior prevalência de insônia $(8,1 \%$ e $4,4 \%$, respectivamente), comparados com o grupo sem SED (1,4\%).

A correlação positiva entre insônia e SED não foi encontrada no estudo de Lickstein et al..23, que utilizaram a escala de sonolência de Stanford e o teste de latências múltiplas do sono. Estes mesmos autores utilizaram-se da pupilometria, outro marcador biológico da SED, e não obtiveram resultados consistentes o suficiente a fim de definir a relação entre SED e insônia ${ }^{24}$.

\section{CONCLUSÃO}

Foi alta a prevalência de SED na população geral de Ribeirão do Largo. Supõe-se que a redução do tempo total de sono, com despertar precoce, provoque a SED no dia seguinte, podendo prejudicar o desempenho das pessoas por ela acometidas. Novos estudos devem ser realizados com critérios e instrumentos cada vez mais amplos e sistematizados para se detectar a prevalência da SED na população geral.

\section{REFERÊNCIAS}

1. Johnson EO, Breslau N, Roehrs T, Chase G, Drake C, Roth T. Epworth and daytime sleepiness sacles: psychometric comparison in a community-based sample. Sleep. 2001;24:A108-9.

2. Ohayon MM, Vecchierini MF, Lubin S. Excessive daytime sleepiness and insomnia in an elderly population. Sleep. 2001;24:A345-6.

3. Stal V, Billaud M, Laaban JP, Lubin S, Iwatsubo Y, Paillard M, et al. Evaluation of the French version of the epworth sleepiness scale in a group of 274 subjects with hypersomnia. Sleep. 2001;24:414-5.

4. Johnson EO, Breslau N, Roth T, Roehrs T, Rosenthal L. Psychometric evaluation of daytime sleepiness and nocturnal sleep onset scales in a representative community sample. Biol Psychiatry. 1999;45(6):764-70.

5. Johns M, Hocking B. Excessive daytime sleepiness. Daytime sleepiness and sleep habits of australian workers. Sleep. 1997;20(10):844-9.

6. Izquierdo-Vicario Y, Ramos-Platón MJ, Conesa-Peraleja D, Lozano-Parra AB. Epworth sleepiness scale in a sample of the spanish population. Sleep. 1997;20(8):676-7.

7. Whitney CW, Enright PL, Newman AB, Bonekat W, Fole D, Quan SF. Correlates of daytime. Sleepiness in 4578 elderly persons: the cardiovascular health study. Sleep. 1998;21(1):27-36.

8. Hara C. Projeto Bambuí: estudo de base populacional de prevalência e fatores associados a sonolência excessiva diurna [dissertação]. Belo Horizonte: Universidade Federal de Minas Gerais, 2001.

9. Hublin C, Kaprio J, Partinen M, Heikkila K, Koskenvuo M. Daytime sleepiness in an adult Finnish population. J Intern Med. 1996;239:417-23.

10. Martikainen N, Urponen H, Partinen M, Hasan J, Vuori l. Daytime sleepiness a risk factor in community life. Acta Neurol Scand. 1992;86:337-41. 
11. Janson C, Gislason T, Backer W, Plaschke P, Björnsson E, Hetta J, et al. Daytime sleepiness, snoring and gastro-esophageal reflux amongst young adult in three European countries. J Intern Med. 1995;237:277-85.

12. Hara C, Rocha FL, Lima-Costa MF. Sonolência excessiva diurna: epidemiologia e impacto. J Bras Psiquiatr. 2002;51(5):313-22.

13. Klink M, Quan SF. Prevalence of reported sleep disturbances in a general adult population and their relationship to obstructive airways diseases. Chest. 1987;91:540-6.

14. Baldwin CM, Griffith KA, Nieto FJ, O'Connor GT, Walsleben JÁ, Redline S. The association of sleep-disordered breathing and sleep symptoms with quality of life in the Sleep Health Study. Sleep. 2001;24(1):96-105.

15. Roberts RE, Shema SJ, Kaplan GA, Strawbridge WJ. Sleep complaints and depression in an aging cohort: a prospective perspective. Am J Psychiatry. 2000;157:81-8.

16. Haraldsson PO, Carenfelt C, Tingvall C. Sleep apnea syndrome symptons and automobile driving in a general population. J Clin Epidemiol. 1992;45(8):821-5.

17. Hays JC, Blazer DG, Foley DJ. Risk of napping: excessive daytime sleepiness and mortally in an older community population. J Am Geriatr Soc. 1996;44:693-8.
18. Newman AB, Spiekerman CF, Enright P, Lefkowitz D, Manolio T, Reynolds CF, et al. Daytime sleepiness predicts mortality and cardiovascular disease in older adults. J Am Geriatric Soc. 2000;48(2);115-23.

19. Associação Americana de Psiquiatria (APA). Manual Diagnóstico e Estatístico de Transtornos Mentais. DSM-IV. $4^{a}$ ed. Porto Alegre: Artes Médicas; 1995. 830p.

20. Ohayon MM, Caulet M, Philip P, Priest RG. How sleep and mental disorders are related to complaints of daytime sleepiness. Arch Intern Med. 1997;157(22):2645-52.

21. Souza JC, Reimão R, Magna LA. Excessive daytime sleepiness in Campo Grande general population, Brazil. Arq Neuropsiquiatr. 2002;60(3):558-62.

22. Roth T, Roehrs TA. Etiologies and sequelae of excessive daytime sleepiness. Clin Ther. 1996;18(4):562-76.

23. Lickstein KL, Wilson NM, Noe SL, Aguillard RN, Bellur SN. Daytime sleepiness in insomnia: behavioral, biological and subjective indices. Sleep. 1994;17:694-702.

24. Lickstein KL, Johnson RS, Gupta SS, O'Laughlin DL, Dykstra TA. Are insomniacs sleepy during the day? A pupillometric assessment. Behave Res Ther. 1992;30:283-92. 